\title{
Intégrines, développement embryonnaire et maladies : avancées récentes
}

es récepteurs intégrines (plus d'une vingtaine d'hétérodimères $\alpha \beta$ actuellement connus), sont les principaux médiateurs des interactions entre les cellules et leur environnement, assurant un lien essentiel entre la matrice extracellulaire (MEC), le cytosquelette intracellulaire, et les voies de transmission du signal. Ces récepteurs interviennent dans de multiples fonctions telles que la prolifération/différenciation, la migration/ adhérence ou l'organisation et le maintien de l'architecture cellulaire et tissulaire. Les approches génétiques développées au cours de ces dernières années chez la souris ont montré le rôle-clé des intégrines dans l'organisme. Récemment, leur participation à des processus aussi divers que la placentation, l'intégrité musculaire, l'intégrité de l'épiderme ou encore le développement du système nerveux central a été démontrée.

\section{Angiogenèse et placentation : rôle des intégrines $\alpha v$}

Cinq intégrines partagent la même chaîne $\alpha_{v}$ (dont $\left.\alpha_{v} \beta 1, \alpha_{v} \beta 3, \alpha v \beta 5\right)$ et lient des ligands de la MEC, comme la fibronectine $(\alpha v \beta 1)$, la vitronectine ( $\alpha v \beta 3$ ), l'ostéopontine, la tenascine ou encore la laminine et le collagène de type IV. L'expression des intégrines $\alpha \mathrm{v}$ par des types cellulaires très variés suggère leur implication non seulement au cours du développement mais aussi chez l'adulte. La participation essentielle de ces intégrines, en particulier d' $\alpha \mathrm{v} \beta 3$, à la formation des vaisseaux a été suggérée dans un système in vitro d'angiogenèse [1]. Au cours du développement, les intégrines $\alpha v \beta$ fonctionnent aussi comme des récepteurs de la fibronectine, $\alpha v \beta 3$ pouvant participer à l'assemblage de ce type de matrice en l'absence des intégrines $\beta 1$ [2]. L'invalidation de la sous-unité $\alpha \mathrm{v}$, qui entraîne l'absence des cinq hétérodimères $\alpha v \beta$, est létale dans $80 \%$ des cas [3], ce qui confirme l'importance physiologique de ces récepteurs. La létalité embryonnaire survient entre 9,5 et 11,5 jours de gestation et est associée à de sévères anomalies de la placentation. La zone labyrinthique du placenta ainsi que les interdigitations entre vaisseaux fotaux et maternels apparaissent peu développées. Les embryons ont un retard de croissance considérable, un important œdème péricardique associé à des altérations cardiaques. Cependant, de manière surprenante, $20 \%$ des embryons homozygotes mutants survivent jusqu'à la naissance, sans grandes anomalies de développement. Le phénotype de ces animaux associe des altérations de la vascularisation cérébrale, d'importantes hémorragies étant présentes très tôt dans le cerveau, et une fente palatine. De manière très inattendue, l'angiogenèse et la vasculogenèse de la plupart des tissus embryonnaires ne semblent pas affectées, contrairement à ce qu'auraient laissé supposer les expériences initiales in vitro. Il est possible que d'autres intégrines, $\alpha 1 \beta 1$ et $\alpha 2 \beta 1$ par exemple, compensent l'absence d' $\alpha v \beta 3$ ce qui expliquerait l'effet modéré de la mutation. L'analyse de double-mutants permettra probablement de valider cette hypothèse.

$\mathrm{Si}$ ces résultats sont particulièrement surprenants pour ce qui est de la relation $\alpha v \beta 3 /$ angiogenèse, ils soulignent néanmoins le rôle de ces récepteurs dans le développement du placenta. Cette observation vient d'être confirmée par l'analyse des mutants $\beta 3^{-/-}$ [4]. Des défauts comparables sont observés dans les cas de prééclampsie responsables de certains avortements, ce qui motivera à l'avenir la recherche d'altérations des intégrines $\alpha v$ dans de telles pathologies.

\section{Physiologie et pathologie musculaire: rôle des intégrines $\alpha 7 \beta 1$ et $\alpha 5 \beta 1$}

L'approche génétique a révélé l'importance de deux intégrines, $\alpha 7 \beta 1$ et $\alpha 5 \beta 1$, dans l'intégrité muscu- laire: en effet, chez la souris, la mutation de l'intégrine $\alpha 7 \beta 1$, récepteur spécifique de la laminine-1 mais également des laminines-2 et -4 (ou mérosines), conduit à une dystrophie musculaire progressive qui débute peu après la naissance [5]. Cette mutation touche plusieurs types de muscles, et affecte principalement les jonctions myotendineuses. Ces défauts ne semblent pas liés à des altérations du complexe dystrophine-dystroglycane/laminine-2. Très récemment, une altération de l'intégrine $\alpha 7$, reliée dans certains cas à une mutation du gène correspondant, a été identifiée chez des patients atteints d'une myopathie congénitale d'étiologie inconnue jusqu'à présent $\left(\mathrm{m} / \mathrm{s} 1998, n^{\circ} 8 / 9\right.$, p. 972).

L'intégrine $\alpha 5 \beta 1$ semble également indispensable au maintien de l'intégrité musculaire [6]. L'absence totale d' $\alpha 5 \beta 1$, tout comme celle de son principal ligand la fibronectine, étant létale, une telle fonction a pu être révélée par l'utilisation de souris chimères, dont les tissus mosaïques permettaient d'analyser les conséquences d'un déficit plus modéré du récepteur. Chez ces souris, des altérations des muscles squelettiques caractérisées par des processus de dégénérescence/régénération des fibres musculaires ont en effet été observées, notamment dans la tête, le thorax et les membres. Les myoblastes déficients en intégrine $\alpha 5$ sont incapables d'adhérer à la fibronectine, et sont plus susceptibles à l'apoptose, qui pourrait aggraver le processus de dégénérescence musculaire sans toutefois en être la cause première. Il semblerait, mais cela reste à confirmer, que $\alpha 7 \beta 1$ et $\alpha 5 \beta 1$ participent à une fonction d'ancrage des fibres musculaires, indispensable dans les régions de tension mécanique, en particulier à la jonction muscle/tendon: ancrage aux laminines des lames basales, qui ferait intervenir $\alpha 7 \beta 1$ et à la fibronectine du tissu interstitiel, par $\alpha 5 \beta 1$. 


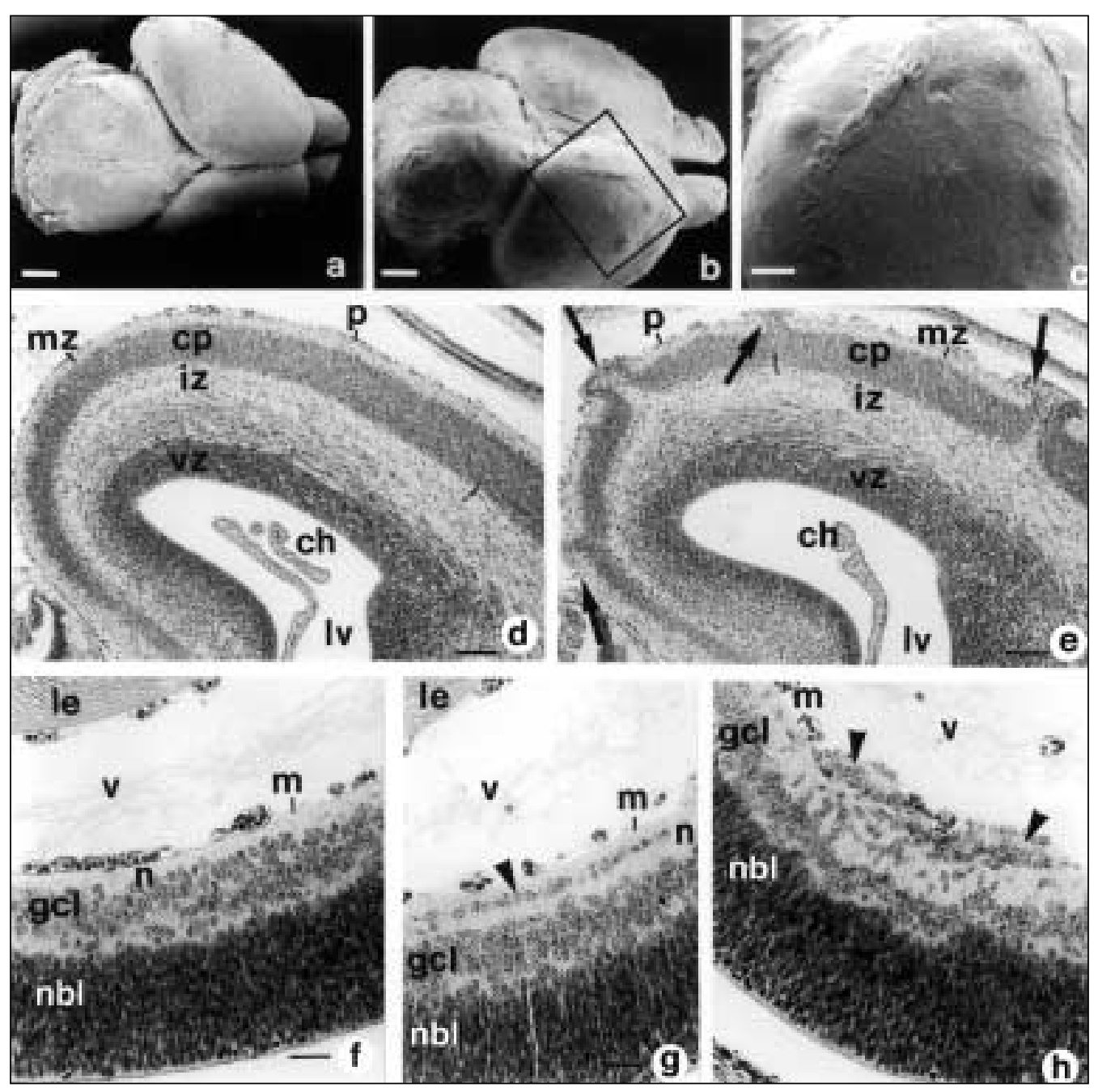

Figure 1. Anomalies morphologiques du cortex cérébral de souris $\alpha 6^{-1-}$ (a-c): ectopies sur la surface cérébrale révélées par microscopie électronique à balayage. (a) cerveau d'un foetus témoin, (b) cerveau d'un fœtus intégrine $\alpha 6^{-/-}$à 18,5 jours, (c) agrandissement de la région encadrée en (b). (d-h): analyse histologique du cortex cérébral et de la rétine. Coupes de cerveaux de fotus (d) témoin, et (e) mutant à 16,5 jours. (d, e) Les flèches montrent une désorganisation de la plaque corticale et des amas de cellules ectopiques dans l'espace sous-arachnoïdien. ( $f$ - $h$ ) Coupes de rétine de foetus (f) témoin et ( $g$, h) mutant à 16,5 jours. $(g, h)$ Les flèches montrent une désorganisation de la couche ganglionnaire de la rétine, et des amas ectopiques dans le corps vitré. ch: plexus choroïde; $c p$ : plaque corticale; gcl: couche ganglionnaire; iz: zone intermédiaire; le: cristallin; Iv: ventricule latéral; $m$ : membrane limitante interne; mz: zone marginale; $n$ : couche de fibres nerveuses; $\mathrm{nbl}$ : couche neuroblastique externe; $p$ : pie-mère; v: corps vitré; vz: zone ventriculaire. Barre = $500 \mu \mathrm{m}$ (a, b), $200 \mu \mathrm{m}(c), 120 \mu \mathrm{m}$ (d, e ), et $30 \mu \mathrm{m}$ (f-h). (Reproduit de la référence [13] avec la permission de Current Biology.)

Jonction dermo-épidermique : rôle de l'intégrine $\alpha 6 \beta 4$

La fonction d'ancrage et de maintien des connexions mécaniques qu'assurent les intégrines est particulièrement critique à la jonction dermo-épidermique. Les modèles animaux reproduisant des altérations de cette jonction se sont révélés extrêmement instructifs pour la caractérisation, chez l'homme, des épidermolyses bulleuses jonctionnelles, maladies de la peau que caractérisent des détachements de l'épiderme souris, les mutations de la chaîne $\alpha 6$ [7] ou de la chaîne $\beta 4[8,9]$, toutes deux s'associant pour former l'hétérodimère $\alpha 6 \beta 4$, sont létales à la naissance, les animaux nouveau-nés présentant un détachement global et massif de l'épiderme. Ce phénotype s'explique par une absence d'hémidesmosomes, complexes jonctionnels dont un des composants essentiels est l'intégrine $\alpha 6 \beta 4$. Des mutations de la laminine-5, ligand d' $\alpha 6 \beta 4$ dans la lame basale, mais également d'autres composants intracellulaires des hémidesmosomes (tels que la plectine et BPAG1) sont aussi respon- sables de phénotypes d'épidermolyses bulleuses [10]. En pathologie humaine, des mutations des gènes codant pour $\alpha 6$ et $\beta 4[11,12]$ ont été identifiées récemment.

Développement du système nerveux central et intégrine $\alpha 6 \beta 1$

La mutation de la sous-unité $\alpha 6$ réalisée dans notre laboratoire a révélé un aspect totalement nouveau et inattendu de la fonction des intégrines dans le système nerveux central [13]. La perte de fonction de cette chaîne 
conduit, en effet, à de sévères anomalies de la rétine et du cerveau (figure 1). La structure laminaire caractéristique de ces deux tissus est très perturbée, et des amas de cellules ectopiques franchissent les barrières limitantes que constituent les lames basales. Ainsi, dans le cortex cérébral, des neuroblastes dont la migration ne s'est pas arrêtée dans la région appropriée de la plaque corticale, traversent la zone marginale, la lame basale et la pie-mère et s'accumulent dans l'espace sous-arachnoïdien (figure $1 \mathrm{~d}$, $e)$. Dans l'œil, de tels amas cellulaires se retrouvent dans le corps vitré (figure $1 f$ - $h$ ). L'hétérodimère $\alpha 6 \beta 1$ est plus probablement en cause que $\alpha 6 \beta 4$, car la sous-unité $\beta 4$ est peu exprimée dans le système nerveux central embryonnaire. Des altérations des dépôts de laminine, ligand spécifique de cette intégrine, ont également été décelées.

Ces résultats illustrent que les récepteurs intégrines et leurs ligands matriciels participent à l'établissement de l'architecture laminaire dans le système nerveux central, et que leur altération peut affecter cette structure. Reste à comprendre si certaines maladies humaines caractérisées par une migration ectopique des neuroblastes pourraient relever d'un semblable mécanisme

\section{RÉFÉRENCES}

1. Brooks PC, Clark RA, Cheresh DA. Requirement of vascular integrin alpha v beta 3 for angiogenesis. Science 1994; 264: 569-71.

2. Wennerberg K, Lohikangas L, Gullberg D, Pfaff M, Johansson S, Fassler R. Beta 1 integrin-dependent and -independent polymerization of fibronectin. I Cell Biol 1996; 132: 227-38.

3. Bader BL, Rayburn H, Crowley D, Hynes RO. Extensive vasculogenesis, angiogenesis, and organogenesis precede lethality in mice lacking all alpha v integrins. Cell 1998; 95 : 507-19.

4. Hodivala-Dilke K, McHugh KP, Tsakiris $\mathrm{DA}$, et al. $\beta 3$-integrin-deficient mice are a model for Glanzmann thrombasthelia showing placental defects and reduced survival. J Clin Inv 1999; 103: 229-38.

5. Mayer U, Saher G, Fassler R, et al. Absence of integrin alpha 7 causes a novel form of muscular dystrophy. Nat Genet 1997; 3: 318-23.

6. Taverna D, Disatnik MH, Rayburn H, et al. Dystrophic muscle in mice chimeric for expression of alpha5 integrin. J Cell Biol 1998; 143: 849-59.

7. Georges-Labouesse E, Messaddeq N, Yehia G, Cadalbert L, Dierich A, Le Meur M. Absence of integrin alpha 6 leads to epidermolysis bullosa and neonatal death in mice. Nat Genet 1996; 13: 370-3.

8. Dowling J, Yu QC, Fuchs E. Beta4 integrin is required for hemidesmosome formation, cell adhesion and cell survival. I Cell Biol 1996; 134: 559-72.

9. van der Neut R, Krimpenfort P, Calafat J, Niessen CM, Sonnenberg A. Epithelial detachment due to absence of hemidesmosomes in integrin beta 4 null mice. Nat Genet 1996; 13: 366-9.
10. Gorski JP, Olsen BR. Mutations in extracellular matrix molecules. Curr Opin Cell Biol 1998; 10 : 586-93.

11. Pulkkinen L, Kimonis VE, Xu Y, Spanou EN, McLean WH, Uitto J. Homozygous alpha6 integrin mutation in junctional epidermolysis bullosa with congenital duodenal atresia. Hum Mol Genet 1997; 6: 669-74.

12. Vidal F, Aberdam D, Miquel C, Christiano AM, Pulkkinen L, Uitto J, Ortonne JP, Meneguzzi G. Integrin beta 4 mutations associated with junctional epidermolysis bullosa with pyloric atresia. Nat Genet 1995; 10 : 229-34.

13. Georges-Labouesse E, Mark M, Messaddeg N, Gansmuller A. Essential role of alpha 6 integrins in cortical and retinal lamination. Curr Biol 1998; 8: 983-6.

\section{Adèle De Arcangelis}

Chercheur post-doctorant.

\section{Élisabeth Georges-Labouesse}

Chargée de recherche au Cnrs.

Institut de génétique et de biologie moléculaire et cellulaire, Cnrs/Inserm/ULP, BP 163, 67404 Illkirch, CU de Strasbourg, France.

\section{TIRÉS À PART}

E. Georges-Labouesse.



